\title{
NON EQUILIBRIUM IONIZATION IN THE LOCAL HOT BUBBLE
}

\author{
M. Arnaud, R. Rothenflug, R. Rocchia \\ Service d'Astrophysique - CEN Saclay -
}

\begin{abstract}
Soft X-ray surveys proved the existence of a local hot bubble surrounding the solar system (Mc Cammon et al 1983). Recently two experiments measured the spectrum of this hot interstellar medium with solid state detectors during rocket flights between 300 and $1000 \mathrm{eV}$ (Rocchia et al 1984). The good resolution of the experiment permitted to measure CV, CVI and OVII line emissivities with good accuracy. We use these data to put some constraints on a model where the emission comes from a supernova remnant expanding in a finite pressure medium (Cox and Anderson 1982). We show the importance of non equilibrium ionization phenomena.
\end{abstract}

\section{INTRODUCTION}

In a recent paper, Rocchia et al (1984) reported spectral observations of the soft Xray background between 0.3 and $1 \mathrm{keV}$ with solid state detectors. One of their spectra is characteristic of the local hot bubble, and concerns a part of the North Galactic hemisphere including the Hercules Hole and the North Galactic pole. This spectrum exhibits features identified with lines of CV-CVI around $300 \mathrm{eV}$ and OVII around $530 \mathrm{eV}$.

In this paper, we use these data to put some constraints on a model where the emission of the soft X-ray background comes from a supernova remnant surrounding the solar system. Such a model was previously developed by Cox and Anderson (1982) in order to reproduce the $X$ flux in the so-called $B$ band ( $130-188 \mathrm{eV})$ and $C$ band (160-284 eV).

2. HYDRODYNAMICAL MODEL AND METHOD FOR COMPUTING THE IONIZATION STRUCTURE

Gaffet (1978) proposed a simple analytical approximation for the hydrodynamical evolution and the structure of a supernova in the case where the external medium pressure is not negligeable with respect to the shock pressure. The model parameters are $E_{o}$, the explosion energy and $n_{0}, T_{0}$ the external electronic density and temperature. In this first approach, we fix the dimension of the remnant to $R_{s}=80 \mathrm{pc}$ which is equivalent, for a given set of parameters, to settle the age of the remnant. This dimension corresponds to the maximum radius we established for the local hot bubble from studies of soft X-ray absorption by an interstellar cloud (Arnaud, Rothenflug 1984a). We also settle the external temperature to $T_{0}=5.10^{5} \mathrm{~K}$ (see Mc Kee, Ostriker 1977).

In order to calculate the X-ray spectrum of such a remnant, the ionization structure must be determined in following the history of each shell from the time it is shocked until the time to be considered. The method we follow is very similar to the method described by Cox and Anderson (1982). The derivatives of each ionization stage of each element were integrated numerically by an implicit method of the Runge-Kutta type. Fifty shells were followed, divided in such a way to obtain a great number of shells near the shock where the conditions vary very quickly. The ionization and recombination rates we used are taken from an up-to dated evaluation we recently made (Arnaud, Rothenflug, 1984b). 


\section{THE SOFT X-RAY SPECTRUM}

We reproduce in figure 1 the soft $X$-ray spectrum of the local hot bubble discussed in Rocchia et al (1984). The contribution of the various components to the total spectrum is depicted in the figure. Instead of calculating the total spectrum with our model, we extracted from the data the intensities of lines corresponding to the $(\mathrm{CV}+\mathrm{CVI})$ blend and to the OVII ion. To do so, we assumed that the continuum in our non equilibrium model will have about the same low intensity than that of figure 1 . Moreover, we do not use $\mathrm{N}$ VI intensity as a constraint, since its intensity is about the same as the continuum intensity.

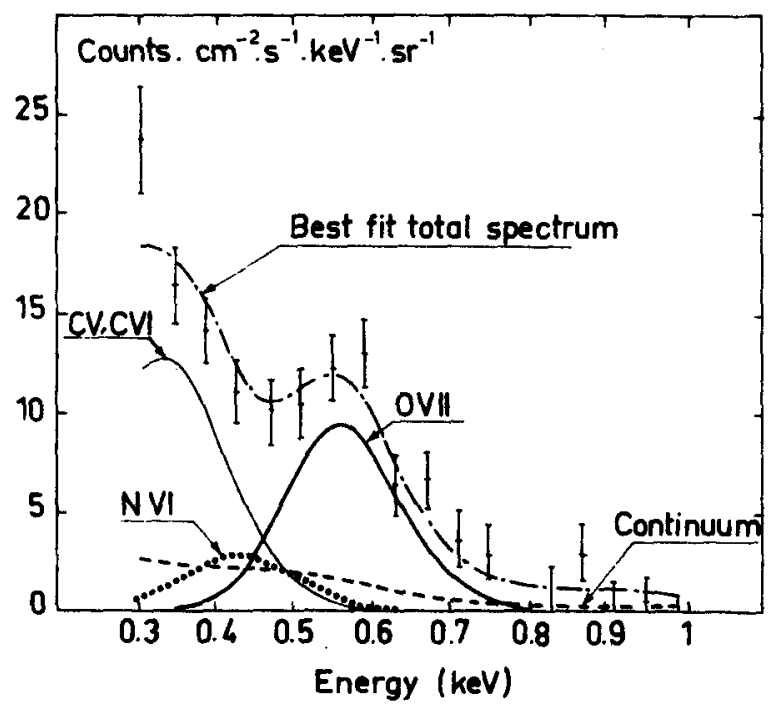

Fig.1 : Contribution of the various ion species to the local hot bubble spectrum (Rocchia et al 1984). The dot-dashed curve is the total spectrum. The dashed curve shows the contribution of the continuum (bremsstrahlung + recombination radiation). Small contributions of several species of $\mathrm{S}, \mathrm{Si}, \mathrm{Fe}$, are not depicted.

To obtain the statistical uncertainties on each line, we followed the method described by Inoue et al (1980). We corrected for the detector sensitivity and for an interstellar absorption of $\sim 6.10^{19} \mathrm{H}$ atoms $/ \mathrm{cm}^{2}$ (Inoue et al (1979). We obtained the following figures :

$C V+C V I \quad 45 \pm 9$ photons $/ \mathrm{cm}^{2}$.s.ster.

O VII : $4 \pm 0.9$ photons $/ \mathrm{cm}^{2}$.s.ster.

(error at the $99 \%$ confidence level).

\section{CONSTRAINTS ON THE MODEL}

Once fixed the supernova remnant radius $R_{s}$ and the external temperature $T_{0}$, two parameters, $E_{o}$ and $n_{0}$ remain free. It is more convenient to use the couple $E_{o}$ and $T_{s}$, where $T_{s}$ is the temperature just behind the shock. Indeed, one can show, from the work of Gaffet, that for a given set of $R_{5}, T_{5}$ and $T_{0}$, both the temperature profile and the reduced density $\left(n(r) / n_{\alpha}\right.$ profile do not depend on $E_{o}$. The ionic fraction profiles do not vary in a so simple way on $\mathrm{E}_{0}$, because the collisionnal ionization and recombination times are proportional to the true value of the density. However in the emissive region, i.e just behind the shock, ionic fractions of CV, CVI and O VII do not strongly depend on $n_{0}$ (and $E_{0}$ ), for a given set of $R_{s}, T_{s}$ and $T_{0}$.

This explains why both the intensities of (CV + CVI) lines and $O$ VII lines are proportional to $n_{0}$ (for $R_{S}, T_{5}, T_{0}$ fixed) and that the intensities of both set of lines 
are proportional. This is illustrated in figure 2 where we plotted the variation of the CV + CVI line intensity in function of the OVII line intensity, for different values of $E_{0}$ and $T_{S}$ (with fixed values of $R_{S}=80 p c, T_{0}=5.10^{5} \mathrm{~K}$ ). The dashed contour delimits the constraints given by our observational data on the: line intensities. The constraints on $\left(E_{0}, T_{s}\right)$ can be transformed in constraints on $\left(E_{0}, n_{0}\right)$ as shown in figure 3 : our data lead to relatively tight constraints on the values of $E_{0}$ and $n_{0}$. A good agreement with the data is reached for $E_{0}=6.10^{50}$ ergs, $n_{0}=1.810^{-2} \mathrm{~cm}^{-3}\left(\mathrm{~T}_{\mathrm{s}}=1.110^{6} \mathrm{~K}\right)$.
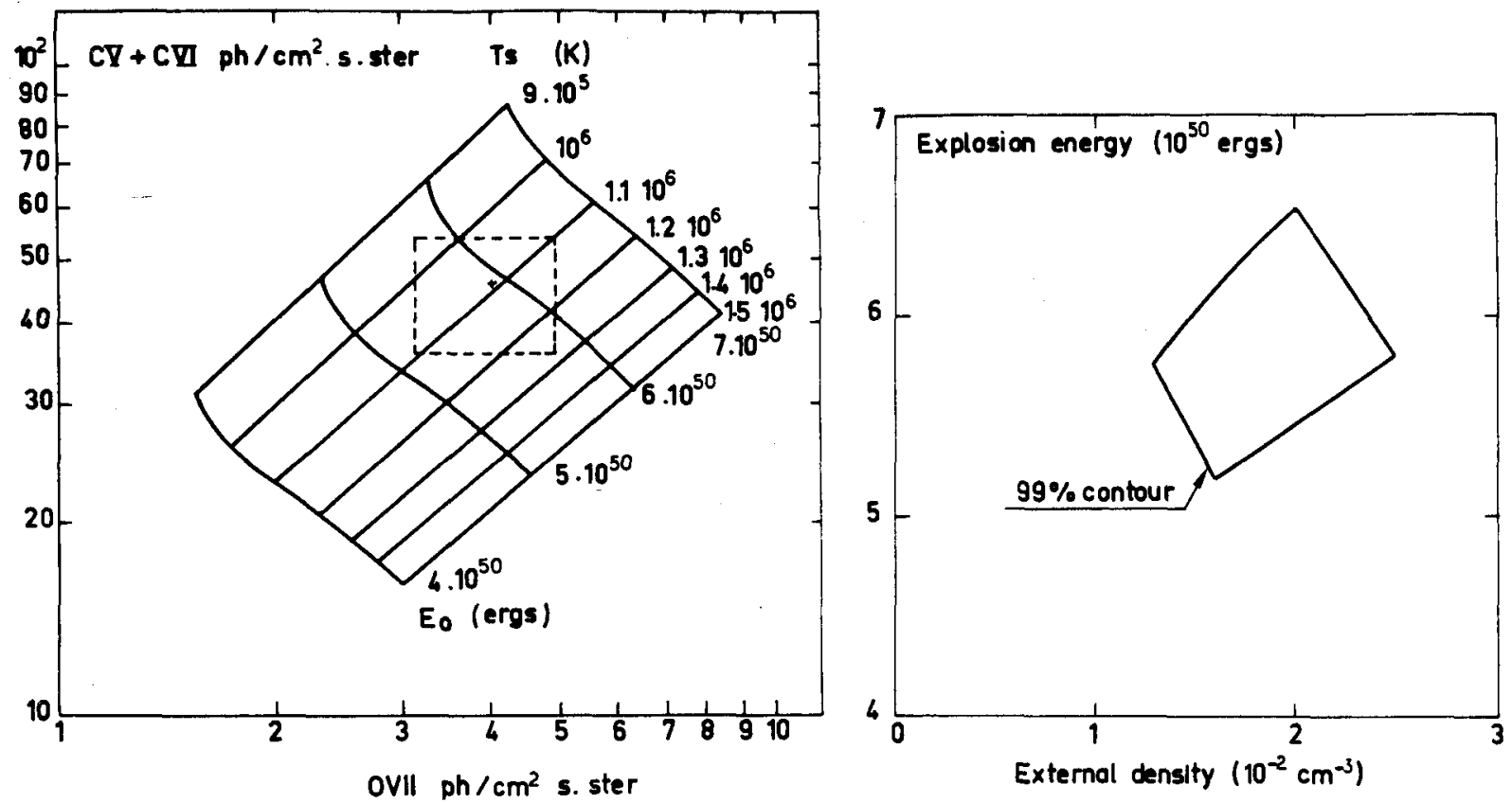

Fig.2 : Full lines: $(\mathrm{CV}+\mathrm{CVI})$ and OVII line Fig.3 : $99 \%$ confidence contour on the intensities computed in our model for parameters.

different values of $\mathrm{E}_{0}$ and $\mathrm{T}_{\mathrm{s}}$. Dashed line :

intensity range allowed by observations.

For this set of parameters, the ionic fraction profiles of $C$ and $O$ ions are depicted in figure 4, in comparison with their values in ionization equilibrium. If the $O$ VII (helium-like ) ion pratically keeps its equilibrium value in the most emissive region, just behind the shock, carbon ions present a strong ionization delay.

In addition, for the range of parameters determined from the X-ray spectroscopic measurements by Rocchia et al (1984), we deduced an OVI volumn density N OVI $=2-7$ $10^{12}$ ions $\mathrm{cm}^{-2}$, quite coherent with the values deduced from UV spectroscopy in the direction of nearby stars (Jenkins 1978). 

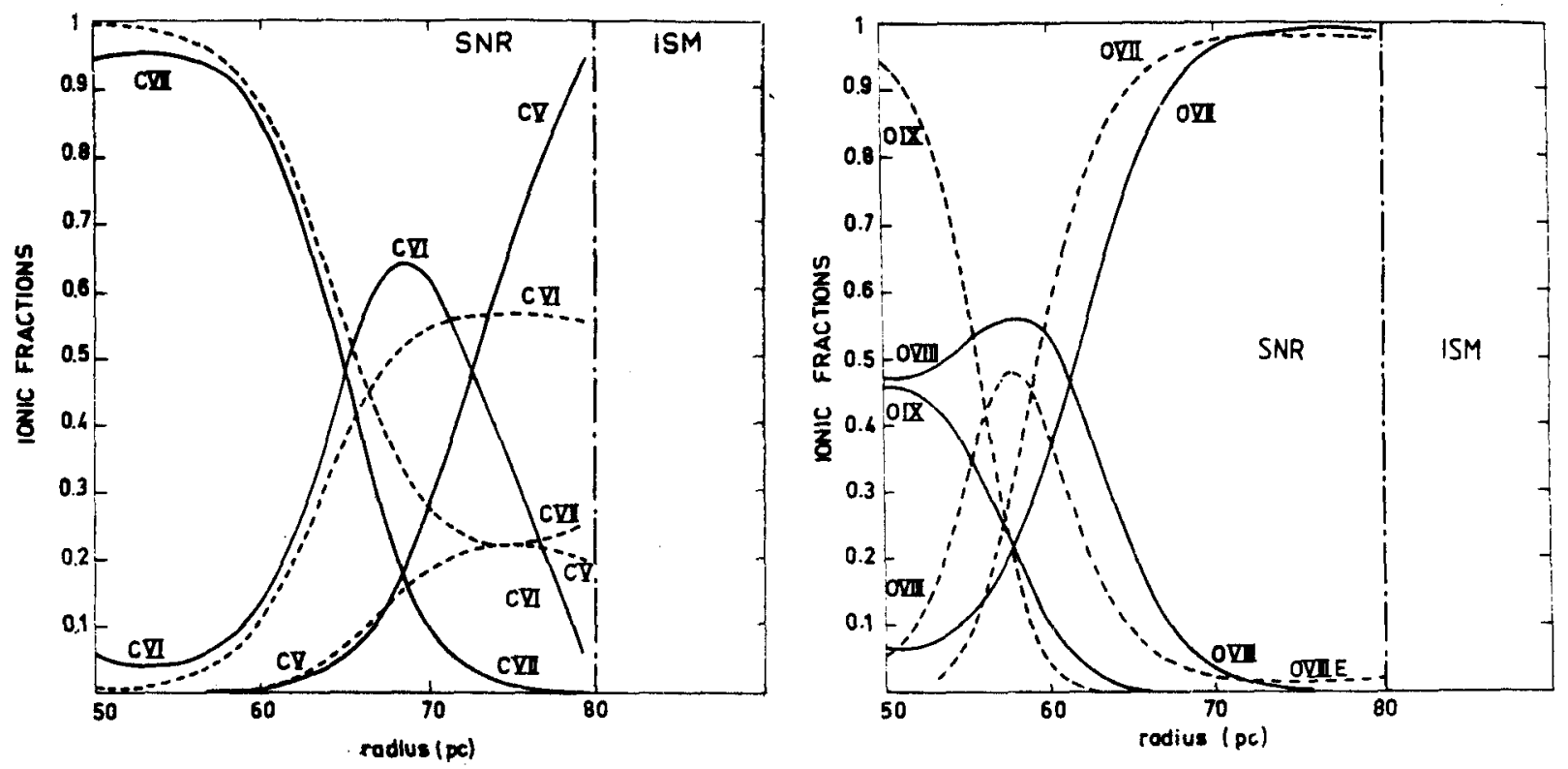

Fig.4 : lonic profiles behind the shock for the set of parameters corresponding to the best fit. Full curve: non-equilibrium values. Dashed curve: equilibrium values

\section{REFERENCES}

Arnaud M., Rothenflug R. 1984a A.A. (in press) $1984 \mathrm{~b} \mathrm{A.A.} \mathrm{(in} \mathrm{press).}$

Cox D.P., Anderson P.R.1982, Ap.J. 253, 268.

Gaffet B. 1978, Ap.J. 225, 442.

Inoue H., et al 1979, Ap.J.(Lett) 227, L85.

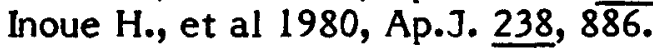

Jenkins E.B., 1978, Ap.J. 219, 845.

MC Cammon D., et al 1983, Ap.J. 269, 107.

Mc Kee C.F., Ostriker J.P., 1977, Ap.J. 218, 148.

Rocchia R., et al 1984, A.A. 130, 53. 